\title{
SPECTRAL ESTIMATES FOR DIRICHLET LAPLACIANS ON PERTURBED TWISTED TUBES
}

\author{
PAVEL EXNER AND DIANA BARSEGHYAN
}

Abstract. We investigate Dirichlet Laplacian in a straight twisted tube of a non-circular cross section, in particular, its discrete spectrum coming from a local slowdown of the twist. We prove a Lieb-Thirring-type estimate for the spectral moments and present two examples illustrating how the bound depends on the tube cross section.

Mathematics subject classification (2010): 81Q37, 35P15, 81Q10.

Keywords and phrases: Dirichlet Laplacian, twisted tube, discrete spectrum, eigenvalue estimates.

\section{REFERENCES}

[1] M. Sh. Birman, G. YE. Skvortsov, On the square summability of the highest derivatives of the solution to the Dirichlet problem in a region with piecewise smooth boundary, Izv. Vyssh. Uchebn. Zaved. Matem. 30 (1962), 12-21 (in Russian).

[2] Ph. Briet, G. RAIKov, H. KovaŘír, E. SocCorsi, Eigenvalue asymptotics in a twisted waveguide, Comm. PDE 34 (2009), 818-836.

[3] I. J. Clark, A. J. BRACKen, Bound states in tubular quantum waveguides with torsion, J. Phys. A: Math. Gen. 29 (1996), 4527-4535.

[4] T. EKholm, H. KovaŘíK, D. KREJČIŘík, A Hardy inequality in twisted waveguides, Arch. Rational Mech. Anal. 188 (2008), 245-264.

[5] P. Exner, H. Kovaří Spectrum of the Schrödinger operator in a perturbed periodically twisted tube, Lett. Math. Phys. 73 (2005), 183-192.

[6] P. EXNER, H. Linde, T. WEIDL, Lieb-Thirring inequalities for geometrically induced bound states, Lett. Math. Phys. 70 (2004), 83-95.

[7] T. Kato, Perturbation Theory for Linear Operators, Classics in Mathematics, Springer-Verlag, Berlin 1995.

[8] A. Laptev, T. WeidL, Sharp Lieb-Thirring inequalities in high dimensions, Acta Math. 184 (2000), $87-100$.

[9] A. Posilicano, On the many Dirichlet Laplacians on a non-convex polygon and their approximations by point interactions, arXiv:1104.5264. 